\title{
Niveles de factor de crecimiento transformante beta-3 y óxido nítrico en cuatro concentrados autólogos de plaquetas y plasma derivados de sangre equina
}

\author{
Transforming growth factor beta-3 and nitric oxide levels in four autologous \\ platelet concentrates and plasma derived from equine blood \\ JU Carmona ${ }^{a^{*},}$ D Argüelles ${ }^{b}$, M Prades $^{\mathrm{b}}$ \\ aDepartamento de Salud Animal, Universidad de Caldas, Manizales, Caldas, Colombia. \\ bDepartamento de Medicina y Cirugía Animal, Universidad Autónoma de Barcelona, Edificio V. 08193. Bellaterra, Barcelona, \\ España.
}

\begin{abstract}
SUMMARY
The aim of this study was to evaluate single and double centrifugation tube methods for concentrating equine platelets, leucocytes, transforming growth factor beta-3 (TGF- $\beta_{3}$ ) and nitric oxide (NO). Whole blood samples were collected from clinically healthy horses and processed using single and double centrifugation tube methods to obtain four platelet concentrates (PCs): PC-A, PC-B, PC-C and PC-D, which were analyzed using a flow cytometry hematology system for haemogram. Concentrations of TGF- $\beta_{3}$ were determined in all the samples by ELISA and NO levels were determined by Greiss reaction. Platelet and leukocyte concentrations for each PC and whole blood were statistically different $(\mathrm{P}<0.05)$. Levels of TGF- $\beta_{3}$ and NO for each PC and plasma were similar. In conclusion, the single and double centrifugation tube methods are reliable for concentrating equine platelets. However, TGF- $\beta_{3}$ levels do not depend of the number of platelets or leucocytes concentrated with both centrifugation tube procedures.
\end{abstract}

Palabras clave: equinos, plaquetas, factor de crecimiento transformante beta-3, óxido nítrico.

Key words: equine, platelet, transforming growth factor beta-3, nitric oxide.

\section{INTRODUCCION}

Se ha propuesto la utilización de concentrados autólogos de plaquetas (PCs) como tratamiento de lesiones traumáticas y degenerativas del aparato locomotor de caballos (Argüelles y col 2005, Carmona y col 2005, Prades y col 2006). Se piensa que la gran cantidad de factores de crecimiento (GFs) liberados a dosis suprafisiológicas, cuando se produce la activación de un PC podría influir de manera positiva sobre la reparación de tejidos previamente lesionados (Marx 2004) e incluso afectados por trastornos degenerativos (Argüelles y col 2005, Carmona y col 2005, Prades y col 2006). Los principales GFs presentes en los gránulos alfa de las plaquetas incluyen los factores de crecimiento derivados de las plaquetas (PDGFs) AA, $\mathrm{AB}$ y $\mathrm{BB}$, factor de crecimiento transformante beta- 1 (TGF- $\beta_{1}$ ), beta-2 (TGF- $\beta_{2}$ ), factor de crecimiento epidérmico (EGF), factor de crecimiento vascular endotelial (VEGF), factor de crecimiento insulínico tipo I (IGF-I) y factor de crecimiento de los hepatocitos (HGF), entre otros (Zimmermann y col 2001).

Los niveles de GFs presentes en concentrados autólogos de plaquetas humanas (Weibrich y col 2005) o equinas (Sutter 2004, Argüelles y col 2006) pueden depender,

Aceptado: 24.07.2007.

* Departamento de Salud Animal, Universidad de Caldas, Calle 65 Nº26-10, Manizales, Caldas, Colombia; carmona@ucaldas.edu.co entre otras cosas, de la técnica usada para concentrar las plaquetas, del número de plaquetas concentradas y del grado de concentración leucocitaria. Hasta el momento, se han descrito cuatro técnicas generales para producir PCs equinos: aféresis (automatizada) (Carter y col 2003), buffy coat (semiautomatizada), buffy coat más filtro (Sutter y col 2004) y centrifugado en tubo (manual) (Argüelles y col 2006). La técnica de centrifugado simple y doble en tubo (Argüelles y col 2006) ha adquirido gran popularidad en clínica equina, ya que es un procedimiento fácil de realizar y es muy económico (Argüelles y col 2005, Carmona y col 2005, Prades y col 2006) en comparación con las demás técnicas (aparatos) usadas para producir PCs en caballos (Carter y col 2003, Sutter y col 2004).

Se han descrito los niveles de plaquetas y leucocitos que pueden ser concentrados con cada técnica en el caballo (Carter y col 2003, Sutter y col 2004, Argüelles y col 2006). También se han documentado los niveles de algunos factores de crecimiento (TGF- $\beta_{1}$, TGF- $\beta_{2}$, PDGF-AB, PDGF-BB e IGF-I) (Carter y col 2003, Sutter y col 2004, Argüelles y col 2006) e incluso se ha descrito el grado de activación de las plaquetas equinas en PCs obtenidos mediante centrifugado en tubo (Argüelles y col 2006). Es necesario considerar que estas investigaciones han permitido explicar, en parte, el efecto terapéutico de los PCs, cuando estos son inyectados en lesiones degenerativas del aparato locomotor de caballos (Argüelles y col 2005, Carmona y col 2005, Prades y col 2006). Sin embargo, 
la investigación celular y molecular de los PCs equinos obtenidos mediante diversas técnicas es aún incipiente, por lo que es necesario realizar nuevos estudios que permitan ampliar este conocimiento.

Según el conocimiento de los autores, no se han reportado los niveles de TGF- $\beta_{3}$ y oxido nítrico (NO) en PCs equinos obtenidos mediante la técnica de centrifugado simple o doble en tubo. El TGF- $\beta_{3}$ es una molécula pleiotrópica presente en numerosos tejidos. Este factor de crecimiento posee efectos antifibróticos y angiogénicos (Theoret y col 2001, Theoret y col 2002). El NO es una molécula de vital importancia en la fisiología endotelial, ya que produce vasodilatación capilar y evita la activación y agregación de las plaquetas. Cuando este gas es producido en exceso, actúa como radical libre con alto poder oxidativo sobre las membranas celulares. Es necesario aclarar que la acción general de esta molécula es muy compleja, ya que entre otros procesos fisiológicos también se encarga de disminuir la respuesta inflamatoria (Schwentker y col 2002).

Los objetivos de esta investigación fueron (a) describir y comparar los niveles de plaquetas y leucocitos, en cuatro concentrados de plaquetas (A, B, C y D) equinos obtenidos mediante la técnica de centrifugado simple y doble en tubo (Argüelles y col 2006) y (b) describir y comparar los niveles de TGF- $\beta_{3}$ y NO en estos cuatro PCs y en plasma equino. Las hipótesis de esta investigación fueron que (a) los niveles celulares, de TGF- $\beta_{3}$ y NO diferían estadísticamente entre cada PC y plasma y que podrían existir correlaciones entre el número de leucocitos y plaquetas de cada PC y los niveles de TGF- $\beta_{3}$ y NO.

\section{MATERIAL Y METODOS}

\section{CABALLOS}

Veintiséis caballos de diferentes razas, clínicamente sanos, fueron incluidos en este estudio, 11 yeguas, 1 semental y 14 machos castrados, con un rango de edad entre 3-12 años (edad media 6,9 \pm 2,3 años). Todos los animales estaban estabulados, bajo condiciones similares de alimentación y manejo, en una escuela de equitación cercana al Hospital Clínico Veterinario de la Universidad Autónoma de Barcelona.

\section{RECOLECCION DE LA SANGRE}

Ciento veinticinco mililitros de sangre entera fueron extraídos de la vena yugular de cada caballo, mediante un catéter mariposa de 23G (Terumo, Leuven, Bélgica); luego fueron depositados en 25 tubos de citrato de sodio al 3,8\% (BD Systems, Vacutainer, Plymouth, Reino Unido). La sangre de un tubo elegido al azar fue utilizada para realizar un hemograma y posteriormente para preparar plasma. Los otros 24 tubos también fueron escogidos al azar para preparar los PCs; 16 tubos $(80 \mathrm{ml})$ fueron usados en el protocolo de centrifugado doble y los 8 tubos restantes fueron empleados en el protocolo de centrifugado simple. El tiempo promedio entre la extracción de la sangre y la iniciación del procesamiento de las muestras fue de 30 minutos.

\section{METODO DE CENTRIFUGADO SIMPLE EN TUBO}

Cuarenta $\mathrm{ml}$ de sangre citratada ( 8 tubos de $5 \mathrm{ml} /$ cada uno) fueron centrifugados (Hettich Rotofix 32, Tuttlingen, Alemania) a $120 \times g$ por 5 minutos. Se obtuvieron dos fracciones diferentes de $\mathrm{PCs}$, las cuales fueron clasificadas arbitrariamente como PC-A y PC-B. El PC-A fue considerado como el $50 \%$ fracción plasmática más cercana a la capa leucocitaria (buffy coat) y el PC-B fue clasificado como el $50 \%$ de la fracción plasmática inmediatamente superior al PC-A (figura 1A). Cada fracción fue obtenida con un aguja espinal No $18 \mathrm{G}$ de 9 centímetros (BD Yale Spinal, Madrid, España), acoplada a una jeringa de plástico de $20 \mathrm{ml}$. Cinco $\mathrm{ml}$ de cada PC fueron depositados por separado en tubos de polipropileno de $10 \mathrm{ml}$ (Deltalab, S.A., Rubí, España).

\section{METODO DE CENTRIFUGADO DOBLE EN TUBO}

Ochenta $\mathrm{ml}$ de sangre citratada (16 tubos de $5 \mathrm{ml}$ cada uno) fueron centrifugados a 120 x $g$ por 5 minutos. Después se obtuvieron $20 \mathrm{ml}$ de la fracción de PC-A a partir de la aspiración de cada tubo, los cuales fueron depositados en dos tubos de polipropileno de $10 \mathrm{ml}$. Esta fracción fue centrifugada de nuevo a 240 x $g$ por 5 minutos. La muestra centrifugada de PC-A (previamente centrifugada) fue dividida arbitrariamente en dos fracciones, PC-C y PC-D. La fracción PC-C fue considerada como la fracción más baja del $25 \%$ de la muestra centrifugada de PC-A. El PC-D fue clasificado como la fracción restante $(75 \%)$ inmediatamente superior al PC-C. (figura 1B). Cinco ml de PC-C y de PC-D fueron depositados por separado en tubos de polipropileno de $10 \mathrm{ml}$ y utilizados en el estudio. El número de fuerzas relativas de centrifugación ( $\mathrm{rfc}$ o $g$ ) y el tiempo de centrifugación empleados en este estudio fueron determinados en ensayos preliminares (datos no mostrados).

\section{RECUENTO DE PLAQUETAS Y LEUCOCITOS}

Se realizó un hemograma completo por duplicado a cada muestra (sangre entera, PC-A, PC-B, PC-C y PC-D). Para ello se utilizó un sistema hematológico de citometría de flujo (ADVIA 120 Analyser, Bayer Lab, NY, USA). Los parámetros evaluados incluyeron volumen de paquete celular (PCV), número de plaquetas y leucocitos.

\section{ACTIVACION DE LOS CONCENTRADOS DE PLAQUETAS}

Después de realizar el hemograma a cada PC, se agregaron $250 \mu \mathrm{l}$ de una solución de cloruro de calcio al 10\% (B 


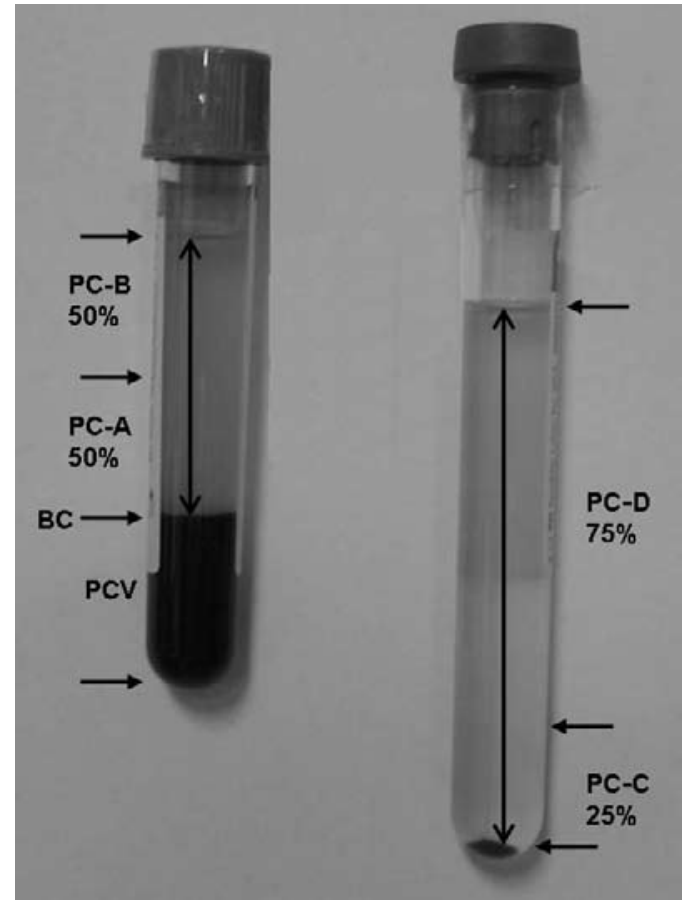

Figura 1. A representa los dos primeros concentrados de plaquetas (PCs) obtenidos mediante el método de centrifugado simple en tubo. B representa los dos concentrados de plaquetas obtenidos mediante el método de centrifugado doble en tubo. $\mathrm{BC}=$ Capa leucoplaquetaria (Buffy coat). $\mathrm{PC}=$ Concentrado de plaquetas. PCV: Volumen de paquete celular.

A represents the first two platelet concentrates (PCs) obtained by the single centrifugation tube method. B represents the second PCs obtained by the double centrifugation tube method. BC = Buffy coat. $\mathrm{PC}=$ Platelet concentrate. PCV: Packed cellular volume.

Braun Medical, Barcelona, España) a $5 \mathrm{ml}$ de cada muestra de PC. Los PCs fueron incubados a temperatura ambiente $\left(20-22{ }^{\circ} \mathrm{C}\right)$ con calcio durante 2 horas para estimular la degranulación de las plaquetas y permitir la formación de coágulos de fibrina. Pasado ese tiempo, el coágulo de fibrina de cada PC fue despegado de las paredes de los tubos y las muestras fueron centrifugadas a $1.720 \mathrm{x} g$ por 8 minutos. El plasma sobrenadante fue obtenido, fraccionado y congelado a $-80^{\circ} \mathrm{C}$, para la posterior determinación de TGF- $\beta_{3}$ y NO. Es necesario aclarar que el tiempo y la temperatura de incubación fueron elegidos según el protocolo descrito por (Zimmermann y col 2001).

\section{DETERMINACION DE LOS NIVELES DE TGF- $\beta_{3}$}

Se determinaron los niveles de TGF- $\beta_{3}$ en los diferentes PCs y plasma de cada caballo. La muestra de plasma fue obtenida mediante la centrifugación de sangre entera citratada a 1.720 x $g$ por 8 minutos. Las concentraciones de TGF- $\beta_{3}$ fueron determinadas mediante una prueba de ELISA en sándwich desarrollada con anticuerpos comerciales para TGF- $\beta_{3}$ humano (Human TGF- $\beta_{3}$; R\&D Systems, Abingdon, Reino Unido). Se utilizó un kit de
ELISA humano para TGF- $\beta_{3}$ ya que se ha encontrado una alta homología para todos los péptidos de la familia TGF- $\beta$ entre mamíferos (Javelaud y Mauviel 2004). Sin embargo, según la literatura revisada, no se ha realizado la secuencialización del TGF- $\beta_{3}$ equino; aunque Theoret y col 2001 y Theoret y col 2002 han empleado previamente el anticuerpo humano en un estudio de piel en caballos.

Ampliamente, la prueba de ELISA fue realizada por duplicado en cada muestra. Se empleó un anticuerpo de captura TGF- $\beta_{3}$ murino antihumano (R\&D Systems (parte 840417), Abingdon, Reino Unido) a una concentración de $2 \mu \mathrm{g} / \mathrm{ml}$. El anticuerpo de detección fue un TGF- $\beta_{3}$ de cabra biotinilado antihumano (R\&D Systems (parte 840418), Abingdon, Reino Unido) en una concentración de $100 \mathrm{ng} / \mathrm{ml}$. La línea patrón fue hecha con TGF- $\beta_{3}$ recombinante humano (R\&D Systems (parte 840419), Abingdon, Reino Unido). El TGF- $\beta_{3}$ de todas las muestras fue activado mediante una solución de ácido acético 2,5 N/urea $10 \mathrm{M}$ y las muestras fueron diluidas 12-24 veces. Los ELISAs fueron revelados con un conjugado de estreptavidina a peroxidasa (R\&D Systems, Abingdon, Reino Unido) y el substrato colorimétrico Fast OPD (Sigma, MO, USA.). La lectura fue realizada mediante un lector de ELISA (Anthos, Cutlek, Madrid, España) a 540 nanómetros. El umbral de detección de TGF- $\beta_{3}$ para esta prueba fue de $30 \mathrm{pg} / \mathrm{ml}$.

\section{DETERMINACION DE LOS NIVELES DE NO}

Se determinaron los niveles de $\mathrm{NO}$ en los diferentes PCs y plasma de cada caballo a través de su producto de degradación, los nitritos $\left(\mathrm{NO}_{2}\right)$, por medio del ensayo de diazotización (reacción de Greiss) previa reducción de los nitratos. Se sabe que el $\mathrm{NO}_{2}$ es un producto final y estable del ON en una cantidad aproximada de 51,5 $\pm 3,5 \%$ del total ON producido (Archer 1993).

Primero se construyó una recta patrón a partir de un estándar de nitrito sódico al $100 \mathrm{mM}$. La recta patrón fue iniciada a partir de una dilución 1/1000 del estándar, para comenzar con una concentración máxima de $100 \mu \mathrm{M}$. Posteriormente, las muestras fueron centrifugadas a 1780 $\mathrm{x} g$ durante 10 minutos y sin diluir fueron colocadas en alícuotas de $100 \mu \mathrm{l}$ en placas de 96 pozuelos (Ref. 353915, BD; NJ, USA). El contenido de cada pozuelo fue mezclado con $100 \mu \mathrm{l}$ de una solución de trabajo, la cual fue fabricada a partir de dos partes iguales de sulfanilamida al 1\% más ácido fosfórico al 5\% y N-1-Napthilethileno diamina (NEDA) al 0,1\%. Cada muestra permaneció 10 minutos bajo incubación a temperatura ambiente y posteriormente fue medida en un lector de ELISA (Anthos, Cutlek, Madrid, España) a una absorbancia de $540 \mathrm{~nm}$. A partir de las absorbancias se calculó la recta patrón y sobre la misma se estableció la concentración de nitritos de las muestras. La sensibilidad del método fue de $1 \mu \mathrm{M}$. Todos los resultados se expresaron como $\mu \mathrm{M}$ (Archer 1993). 


\section{ANALISIS ESTADISTICO}

Los datos obtenidos fueron analizados con el programa estadístico SPSS 15.0 (SPSS inc., Il, USA). Los valores del recuento de plaquetas, leucocitos y TGF- $\beta_{3}$ fueron analizados mediante pruebas no paramétricas, ya que estos datos no presentaron una distribución normal (prueba de Shaphiro-Wilk $\mathrm{P}<0,05)$. Estos resultados fueron presentados descriptivamente como medianas con sus respectivos rangos. Los niveles de plaquetas y leucocitos para cada PC y sangre entera citratada fueron analizados mediante una prueba Kruskal-Wallis. Se estableció que en el caso de hallar diferencias estadísticamente significativas se realizarían comparaciones pareadas no paramétricas de Wilcoxon. Los valores de TGF- $\beta_{3}$ exhibieron una gran proporción de datos censurados con desviación a la izquierda, por lo que fue necesario realizar una prueba de Chi ${ }^{2}$ para conocer si existían diferencias estadísticamente significativas relacionadas con una mayor proporción de detección de niveles TGF- $\beta_{3}$ en cada PC y plasma (niveles superiores (detectables) o inferiores a $30 \mathrm{pg} / \mathrm{ml}$ ). Por otra parte, la comparación de los niveles de TGF- $\beta_{3}$ para cada PC y plasma fue realizada mediante la prueba generalizada de Wilcoxon. Se estableció que en el caso de hallar diferencias estadísticamente significativas se realizarían comparaciones pareadas no paramétricas de Wilcoxon.

Los valores de NO presentaron una distribución paramétrica (prueba de Shaphiro-Wilk P >0,05), por lo que fueron presentados como medias con sus respectivas desviaciones típicas. Estos datos fueron analizados mediante un ANOVA de una vía. Se estableció que en el caso de hallar diferencias estadísticamente significativas se realizaría una prueba post-hoc de Tuckey. De manera general se hizo un análisis de correlaciones entre las variables evaluadas para cada PC y plasma. Un valor de $\mathrm{P}<0,05$ fue aceptado como estadísticamente significativo para todas las pruebas.

\section{RESULTADOS}

HEMOGRAMA

Los valores del PCV fueron estadísticamente diferentes $(\mathrm{P}<0,05)$ entre las muestras de sangre entera y cada PC y también entre cada PC. Los PCs obtenidos mediante centrifugado simple o doble presentaron aumentos significativos $(\mathrm{P}<0,05)$ en el recuento de las plaquetas comparados con las muestras de la sangre entera. Los recuentos de plaquetas fueron estadísticamente diferentes $(\mathrm{P}<0,05)$ para cada $\mathrm{PC}$. El PC-C presentó un mayor recuento plaquetario promedio (cuadro 1). El recuento de leucocitos fue estadísticamente diferente para cada PC y sangre entera $(\mathrm{P}<0,05)$. Los recuentos más altos de leucocitos fueron encontrados en el PC-C (cuadro 1).

\section{NIVELES DE TGF- $\beta_{3}$}

Unicamente se detectaron niveles de TGF- $\beta_{3}$ superiores a $30 \mathrm{pg} / \mathrm{ml}$ en el 18,4\% de las muestras evaluadas. El $81,6 \%$ de las muestras restantes presentó niveles de TGF- $\beta_{3}$ inferiores a $30 \mathrm{pg} / \mathrm{ml}$. No se encontró ninguna diferencia estadísticamente significativa (prueba de $\mathrm{Chi}^{2}$ ) en las proporciones de TGF- $\beta_{3}$ detectado (niveles de TGF- $\beta_{3}$ inferiores o superiores a $30 \mathrm{pg} / \mathrm{ml}$ ) entre cada PC y plasma. Tampoco se encontró ninguna diferencia estadísticamente significativa (prueba generalizada de Wilcoxon) entre los niveles de este factor de crecimiento para cada PC y plasma (cuadro 1).

\section{NIVELES DE NO}

El NO fue detectado en el $100 \%$ de las muestras evaluadas. Sin embargo, no se hallaron diferencias estadísticamente significativas entre los niveles de NO para cada PC y plasma (cuadro 1).

Cuadro 1. Valores del hemograma, factor de crecimiento transformante beta-3 y óxido nítrico.

Values for haemogram, transforming growth factor beta-3 and nitric oxide.

\begin{tabular}{|c|c|c|c|c|c|}
\hline \multirow{2}{*}{ Variables } & \multirow{2}{*}{ Sangre entera } & \multicolumn{2}{|c|}{ Centrifugado simple } & \multicolumn{2}{|c|}{ Centrifugado doble } \\
\hline & & PC-A & PC-B & PC-C & PC-D \\
\hline $\operatorname{VPC}(\%)^{*}$ & $27(22-36) a$ & $0(0-0,5) b$ & $0,1(0-0,2) \mathrm{c}$ & $0,3(0-0,9) \mathrm{d}$ & $0(0-0,2) \mathrm{e}$ \\
\hline Plaquetas $\left(\mathrm{CF} \times 10^{3} / \mu \mathrm{l}\right) *$ & $156(91-276) \mathrm{a}$ & $230(127-365) b$ & $229(124-359) \mathrm{c}$ & $273(139-480) d$ & $190(111-294) \mathrm{e}$ \\
\hline Leucocitos (Células x 103/ $/ \mu \mathrm{l}) *$ & $\begin{array}{c}7(5,1-11) a \\
\text { Plasma }\end{array}$ & $4(3,5-5) b$ & $3(1,7-5,1) \mathrm{c}$ & $8,5(3,5-18,3) \mathrm{d}$ & $0,9(0,6-2,5) \mathrm{e}$ \\
\hline $\mathrm{TGF}-\beta_{3}(\mathrm{pg} / \mathrm{ml})^{*}$ & $<30(<30-772,1)$ & $<30(<30-1083,9)$ & $<30(<30-1027,4)$ & $<30(<30-774)$ & $<30(<30-1169)$ \\
\hline Óxido nítrico $(\mu \mathrm{M})^{* *}$ & $33,8(9)$ & $37,1(12,5)$ & $34(10)$ & $35(8)$ & $35(11)$ \\
\hline
\end{tabular}

$\mathrm{CF}=$ Fragmento citoplásmico $; \mathrm{PC}=$ Concentrado de plaquetas; TGF- $\beta_{3}=$ Factor de crecimiento transformante beta-3; VPC $=$ Volumen de paquete celular.

* Los valores son presentados como medianas y rangos.

** Los valores son presentados como medias y desviación estándar.

a,b Valores con letras diferentes denotan una diferencia estadísticamente significativa $(\mathrm{P}<0,05)$. 
De manera general, no se encontró ninguna correlación estadísticamente significativa entre todos los parámetros evaluados.

\section{DISCUSION}

El número de plaquetas, leucocitos y los valores de VPC para cada PC y sangre entera citratada fueron parecidos a los valores descritos previamente por Argüelles y col (2006), lo cual indica que la técnica de centrifugado simple y doble en tubo es reproducible con gran confiabilidad.

Según la literatura revisada, no se han descrito los niveles plasmáticos de TGF- $\beta_{3}$ en el caballo y tampoco se ha reportado la concentración de este péptido en concentrados de plaquetas equinas. Los resultados obtenidos en este estudio demostraron que en la mayoría de los caballos evaluados $(\sim 80 \%-n: 21)$ los niveles plasmáticos de TGF- $\beta_{3}$ presentaron una concentración inferior a $30 \mathrm{pg} / \mathrm{ml}$. Es importante considerar que el $\sim 20 \%$ (n:5) de los caballos restantes era clínicamente normal y no fue posible encontrar una razón que pudiera explicar concentraciones superiores a $30 \mathrm{pg} / \mathrm{ml}$ e incluso tan altas como 772,1 pg/ml. En un estudio en seres humanos sanos se encontró que los niveles plasmáticos de TGF- $\beta_{3}$ eran demasiado bajos ( $<100 \mathrm{pg} / \mathrm{ml}, n: 42)$ (Wakefield y col 1995), mientras que en otro estudio (Jamdar y col 2006), en personas sanas, se observó un valor mediano de TGF- $\beta_{3}$ de $120 \mathrm{pg} / \mathrm{ml}$ (rango: 0-1500, $n: 52$ ). Unicamente 4 caballos $(15 \%)$ de este estudio presentaron niveles de TGF- $\beta_{3}$ entre $30-120 \mathrm{pg} / \mathrm{ml}$. Desde el punto de vista comparativo se podría pensar que los valores plasmáticos de TGF- $\beta_{3}$ en los caballos evaluados son normales. Sin embargo, es necesario aclarar que una de las principales limitantes de este estudio fue no contar con una prueba de ELISA que permitiera detectar niveles de TGF- $\beta_{3}$ inferiores a $30 \mathrm{pg} / \mathrm{ml}$. No obstante, desde el punto de vista fisiológico se ha observado en seres humanos que la concentración plasmática de este péptido, así como la del TGF- $\beta_{2}$ es muy baja $(<5 \%)$, en comparación con la concentración del TGF- $\beta_{1}$ (Wakefield y col 1995). Tal como ha sido observado en personas sanas, la concentración plasmática del TGF- $\beta_{3}$ no está correlacionada con ningún tipo de célula sanguínea (Wakefield y col 1995, Jamdar y col 2006). Esta misma observación pudo ser corroborada en los caballos de este estudio. De manera general se podría aceptar que en el caballo los niveles de TGF- $\beta_{3}$ no dependen de la concentración de plaquetas o de los leucocitos y que el plasma mantiene una concentración constante, normalmente inferior a $30 \mathrm{pg} / \mathrm{ml}$ en caballos sanos.

Los niveles plasmáticos del NO de los caballos de este estudio coinciden con los valores reportados para ponis sanos (Hubert y col 2003). Se decidió evaluar y comparar las concentraciones de NO en los 4 PCs y en plasma, ya que esta molécula podría indicar de manera indirecta una activación inflamatoria de los leucocitos, especialmente de los macrófagos, o un daño celular inducido durante el proceso de centrifugación de las muestras (Schwentker y col 2002). Sin embargo, los niveles de NO no difirieron entre los PCs y el plasma, lo que indica que el protocolo de centrifugado empleado en esta técnica, posiblemente, no induce lesiones o produce estímulos que generen respuestas inflamatorias en los leucocitos centrifugados. Estos resultados complementan las observaciones realizadas por Argüelles y col (2006), quienes demostraron que el volumen promedio de las plaquetas (MPV) no se ve alterado por el protocolo de centrifugación. Cabe aclarar que el MPV es un parámetro que indica activación de las plaquetas en seres humanos y posiblemente también está correlacionado con la activación de las plaquetas en el caballo (Argüelles y col 2006). Otro hecho importante encontrado en este estudio radica en que no se encontró ninguna correlación estadísticamente significativa entre los diferentes componentes celulares y los niveles de NO, lo cual podría indicar que el NO presente en las muestras evaluadas no provenía de las células centrifugadas.

Es importante aclarar que el NO juega un importante papel inmunomodulador y la presencia de este gas es necesaria para una adecuada cicatrización y resolución de la inflamación (Schwentker y col 2002). Es posible que el NO presente en los PCs juegue un importante papel terapéutico, ya que su presencia, entre otras cosas, es necesaria para la activación de las formas latentes de TGF- $\beta_{1}$ a su forma biológicamente activa.

En conclusión, algunas de las hipótesis propuestas en esta investigación pudieron ser corroboradas, e incluso recomprobadas. Esto porque en realidad y, tal como fue observado previamente por Argüelles y col 2006, los niveles celulares de los 4 PCs y la sangre entera difirieron estadísticamente según el protocolo de centrifugado simple o doble en tubo; lo cual, como fue previamente mencionado, indica una gran reproducibilidad de la técnica evaluada. Sin embargo, los niveles de TGF- $\beta_{3}$ y NO no difirieron entre los 4 PCs evaluados y el plasma, lo que indica que no existe ninguna correlación entre estas moléculas y las células presentes en los PCs. Sin embargo, cabe aclarar que los PCs pueden ser considerados como poderosas sustancias biológicas con proporciones adecuadas de sus moléculas concentradas. De esta manera, la investigación deberá continuar, con el objetivo de conocer la cantidad y proporción de otros componentes celulares y moleculares de los PCs, que posiblemente están implicados con el aparente efecto regenerativo de estas sustancias en patologías crónicas del aparato locomotor equino (Argüelles y col 2005, Carmona y col 2005, Prades y col 2006).

\section{RESUMEN}

El objetivo de este estudio fue evaluar el método de centrifugado simple y doble en tubo para concentrar plaquetas, leucocitos, factor de crecimiento transformante beta-3 (TGF- $\beta_{3}$ ) y óxido nítrico (NO). Se recogieron muestras de sangre entera de caballos clínicamente normales, las cuales fueron procesadas por medio del método de centrifugado simple y doble en tubo para obtener 4 concentrados de plaquetas (PCs): PC-A, 
PC-B, PC-C y PC-D. Los PCs y la sangre entera fueron analizados para hemograma mediante un sistema hematológico de citometría de flujo. Las concentraciones de TGF- $\beta_{3}$ fueron analizadas mediante ELISA y el NO mediante la reacción de Greiss. Las concentraciones de plaquetas y leucocitos fueron estadísticamente diferentes $(\mathrm{P}<0,05)$ para cada $\mathrm{PC}$ y sangre entera. Los niveles de TGF- $\beta_{3}$ y NO fueron similares para cada PC y sangre entera. En conclusión, el método de centrifugado simple y doble en tubo es un procedimiento de confianza para concentrar plaquetas en el caballo. Sin embargo, los niveles de TGF- $\beta_{3}$ y NO no dependen del número de plaquetas o de leucocitos concentrados con esta técnica.

\section{AGRADECIMIENTOS}

Los autores desean agradecer el apoyo técnico de Antoni Iborra, Laia Viñals y Paz Martínez del Instituto de Biotecnología y Biomedicina de la Universidad Autónoma de Barcelona, Barcelona, España. También agradecen la valiosa colaboración de Catalina López, MVZ.

\section{REFERENCIAS}

Archer S. 1993. Measurement of nitric oxide in biological models. FASEB J 7, 340-360.

Argüelles D, JU Carmona, F Climent, M Prades. 2005. Platelet rich plasma as a treatment of tendon and ligament injuries in the horse. Proceedings of the $14^{\text {th }}$ Annual Scientific Meeting of the European College of Veterinary Surgeons, Lyon, France, Pp 217-222.

Argüelles D, JU Carmona, J Pastor, A Iborra, L Vinals, P Martinez, E Bach, M Prades. 2006. Evaluation of single and double centrifugation tube methods for concentrating equine platelets. Res Vet Sci 81, 237-245.

Carmona JU, D Argüelles, F Climent, M Prades. 2005. Platelet rich plasma diminishes joint distention and lameness degree in horses with joint disease. $14^{\text {th }}$ Annual Meeting European College of Veterinary Surgeons, Lyon, France, Pp 68-71.

Carter CA, DG Jolly, CE Worden, DG Hendren, CJ Kane. 2003. Plateletrich plasma gel promotes differentiation and regeneration during equine wound healing. Exp Mol Pathol 74, 244-255.

Hubert JD, TL Seahorn, TR Klei, G Hosgood, RM Moore. 2003. Temporal effects of freezing on plasma nitric oxide concentrations in ponies. Can J Vet Res 67, 72-4.
Jamdar S, AF Al-Mowallad, S Kumar, AK Siriwardena. 2006. Differential kinetics of plasma CD105 and transforming growth factor beta expression early in human acute pancreatitis. Pancreas 32, 152-8.

Javelaud D, A Mauviel. 2004. Mammalian transforming growth factorbetas: Smad signaling and physio-pathological roles. Int J Biochem Cell Biol 36, 1161-1165.

Marx RE. 2004. Platelet-rich plasma: evidence to support its usage. J Oral Maxillofac Surg 62, 489-496.

Prades M, I Abellanet, JU Carmona, D Argüelles, M Masri. 2006. Platelet rich plasma: a realistic alternative in tissue repair. $15^{\text {th }}$ Annual Meeting European College of Veterinary Surgeons, Seville, Spain, Pp 211-216.

Schwentker A, Y Vodovotz, R Weller, TR Billiar. 2002. Nitric oxide and wound repair: role of cytokines? Nitric Oxide 7, 1-10.

Sutter WW, AJ Kaneps, AL Bertone. 2004. Comparison of hematologic values and transforming growth factor- $\beta$ and insulin-like growth factor concentrations in platelets concentrates obtained by use of buffy coat and apheresis methods from equine blood. Am J Vet Res 65, 924-930.

Theoret CL, SM Barber, TN Moyana, JR Gordon. 2001. Expression of transforming growth factor $\beta 1, \beta 3$, and basic fibroblast growth factor in full-thickness skin wounds of equine limbs and thorax. Vet Surg 30, 269-77.

Theoret CL, SM Barber, TN Moyana, JR Gordon. 2002. Preliminary observations on expression of transforming growth factors $\beta 1$ and $\beta 3$ in equine full-thickness skin wounds healing normally or with exuberant granulation tissue. Vet Surg 31, 266-73.

Wakefield LM, JJ Letterio, T Chen, D Danielpour, RS Allison, LH Pai, AM Denicoff, MH Noone, KH Cowan, JA O'Shaughnessy, MB Sporn. 1995. Transforming growth factor-beta1 circulates in normal human plasma and is unchanged in advanced metastatic breast cancer. Clin Cancer Res 1, 129-36.

Weibrich G, WK Kleis, WE Hitzler, G Hafner. 2005. Comparison of the platelet concentrate collection system with the plasma-rich-ingrowth-factors kit to produce platelet rich plasma: a technical report. Int J Oral Maxillofacial Implants 29, 118-123.

Zimmermann R, R Jakubietz, M Jakubietz, E Strasser, A Schlegel, J Wiltfang, R Eckstein. 2001. Different preparation methods to obtain platelet components as a source of growth factors for local application. Tranfusion 41, 1217-1224. 